\title{
DFT study of "unlayered graphene solid" formation, in liquid carbon droplets at low pressures
}

\author{
Chathuri Silva ${ }^{1}$ (i) $\cdot$ Philip Chrostoski $^{1} \cdot$ P. Fraundorf ${ }^{1,2}$
}

Received: 14 December 2020 / Accepted: 1 February 2021 / Published online: 18 February 2021

(c) The Author(s) 2021

\begin{abstract}
This atomistic modeling study is companion to new experimental work on carbon vapor slow-cooled in (a) the laboratory and (b) the atmosphere of ancient carbon-synthesizing stars. It specifically follows up on TEM clues about the nucleation of unlayered graphene sheets in a solidifying carbon liquid, to show that 5 atom loops may help explain evidence for faceted pentacones in a slow-cooled melt. This is also first in a series of modeling studies that may open the door to laboratory studies of (i) condensation in cool-giant star atmospheres and (ii) liquid carbon at low pressures.
\end{abstract}

Keywords Computation/computing $\cdot$ Simulation $\cdot$ Extreme environment $\cdot$ Graphene $\cdot$ Liquid $\cdot$ Nucleation \& growth

\section{Introduction}

Circumstellar dust in the laboratory [1-4] is providing insight into materials physics and nuclear physics [5, 6], as well as the astrophysical processes by which such dust is made. Elemental carbon below 100 atmospheres on heating above $3900 \mathrm{~K}$ sublimates to vapor, so liquid carbon is seldom considered to play a role at low pressures even though quenched carbon droplets have been reported in laboratory laser ablation studies [7, 8]. The high-density (KFC1) subset of presolar micron-sized carbon spheres extracted from Murchison meteorite, with isotopic signatures of nucleosynthesis in late stage red giant stars suggesting that they likely condensed in or just outside the hydrogen-rich photosphere at between $10^{-3}$ and $10^{-5}$ atmospheres pressure, have spherical cores that show diffraction rings from randomly oriented atom-thick graphene sheets. Their "graphene-core"/graphite-rim structure likely formed around super-cooled carbon

Supplementary Information The online version of this article (https://doi.org/10.1557/s43580-021-00022-3) contains supplementary material, which is available to authorized users.

Chathuri Silva

ccsq44@mail.umsl.edu

1 Department of Physics and Astronomy, University of Missouri-St. Louis, One University Blvd, Saint Louis, MO 63121, USA

2 Washington University, Saint Louis, MO, USA droplets (diameter in the $300-700 \mathrm{~nm}$ range) that nucleated graphene sheets on randomly oriented pentagonal loops (5 loops) [9]. Laboratory synthesis of carbon particles in a turbo-pumped vacuum evaporating carbon oven also creates such core-rim (and core-only) particles, but graphene sheet coherence widths are much smaller (around $1 \mathrm{~nm}$ ). The lab-grown cores have diameters in the $100-400 \mathrm{~nm}$ range $[10,11]$.

Selected area electron powder diffraction patterns of these cores show only $(h k 0)$ spacings with the high frequency tails expected for atom-thick sheets, and the strange absence of any graphite (002) "layering" lines [10], suggest that they contain unlayered graphene sheets with $4 \mathrm{~nm}$ coherence width [10]. This is reinforced in high-resolution transmission electron microscope (HRTEM) images [9] (cf. Fig. 1) by the presence of intersecting line segments pairs $2-5 \mathrm{~nm}$ in length with "redirection angles" between $39^{\circ}$ and $65^{\circ}$, but no evidence of adjacent "parallel layer" sheets. These intersections suggest that some of the randomly oriented graphene sheets take the form of faceted pentacones, as though they were nucleated on pentagonal loops during solidification $[9$, $10]$.

Additional recent results include the following observations: (i) analysis of electron diffraction patterns from the submicron cores of such particles [12] indicates a diffusescattering carbon matrix of which, in the presolar case about $40 \%$ is unlayered graphene sheets with a mass weighted average size of about 600 atoms, but so far in lab-grown cores is only about $12 \%$ graphene with a mass weighted 


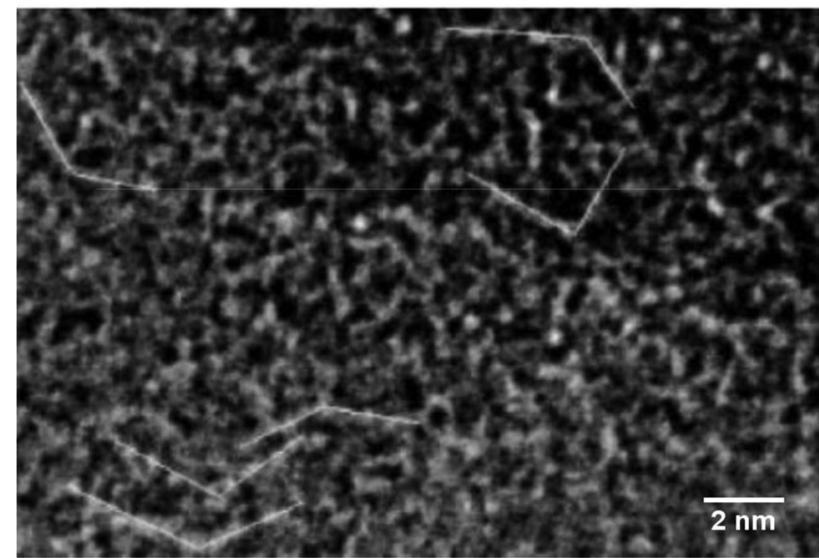

Fig. 1 HRTEM negative of disordered carbon "speckle" and edge-on graphene sheets, in a microtomed (sliced) presolar core, with likely intersecting graphene sheets marked. Adapted with permission from [9]

average size of say 60 atoms, and (ii) atomistic and thermodynamic models of nucleation and growth suggest that laboratory specimens solidified in the $3000 \mathrm{~K}$ range over millisecond times, while the presolar cores solidified at lower temperatures (closer to $2500 \mathrm{~K}$ ) over longer (e.g., $10 \mathrm{~s}$ ) times [13]. These times are still consistent with the near-atomic sharpness of observed the core/rim interfaces, and much shorter than the time for ejection by radiation pressure from a stellar atmosphere.

We show here that density functional theory (DFT) studies with "Vienna ab initio simulation package" (VASP) suggest that 5 loops compete favorably with 6 loops (hexagons) as nucleation seeds for the graphene sheets consistent with previous studies. These studies, still in their infancy, are opening the door to low pressure studies of liquid carbon, and to laboratory studies of carbon condensation in asymptotic giant branch star atmospheres.

\section{Computational methods}

Atom-position relaxations reported here use carbon in a $1.8 \mathrm{~g} / \mathrm{cm}^{3}$ supercell because experimental observations on sliced presolar cores (with graphite rims) appear to have densities on that order $[14,15]$. The relaxations were performed in a supercell using the Projector-Augmented-Wave (PAW) method [16] and generalized gradient approximation (GGA) for exchange correlation functional [17]. We assume low pressure and relax randomized positions of liquid-like $13,20,30,40,60$, and 100-carbon atom clusters in a supercell. This supercell relaxation was using the ISIF 2 tag in

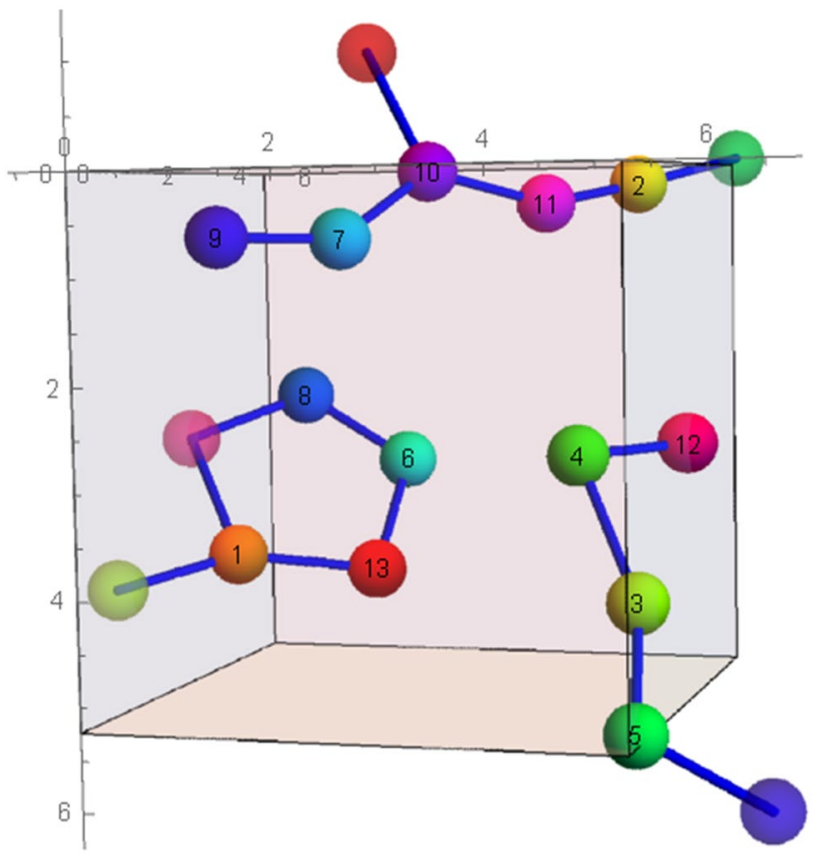

Fig. 2 Covalent bond visualization where $1.7 \AA$ as the covalent bond length cut-off (13 atom set)

VASP in order to keep the periodic boundary conditions in a constant density $1.8 \mathrm{~g} / \mathrm{cm}^{3}$ of carbon in a constant volume and did 20 relaxations for each size of atom sets to obtain the results. The analyzed atom lists follow VASP relaxation of initially random collections with interatom spacings greater that $1.9 \AA$. The volume and the shape of the cell remained as a constant before and after the relaxations and contain six subgroups of results according to the number of atoms in each set (and the supercell volume), are 13 atom sets (144.04 $\left.\AA^{3}\right), 20$ atom sets $\left(221.55 \AA^{3}\right), 30$ atom sets $\left(332.41 \AA^{3}\right), 40$ atom sets $\left(443.21 \AA^{3}\right), 60$ atom sets $\left(664.81 \AA^{3}\right), 100$ atom sets $\left(1108.02 \AA^{3}\right)$.

Another calculation we conducted was 're-relaxing' the atom sets which are previously relaxed using the Longrange Carbon Bond Order Potential (LCBOP) model [18]. To employ the LCBOP model, we used the Large-scale Atomic/Molecular Massively Parallel Simulator (LAMMPS) molecular dynamics program from Sandia National Labs. In this calculation we compare the loops counts, total energies, coordination numbers etc. to see if lower-energy local minima might be obtained thereby. LCBOP and other potentials are also being used to model later stage nucleation and growth of graphene sheets from the liquid melt, but these topics are not the primary goal of this paper. However, detailed re-relaxation results can be found in the Supplementary Material: Sect. 2. 


\begin{tabular}{l|l} 
atoms/loop & \#loops \\
\hline 3 & 100 \\
4 & 50 \\
5 & 92 \\
6 & 48 \\
7 & 89
\end{tabular}

(a)

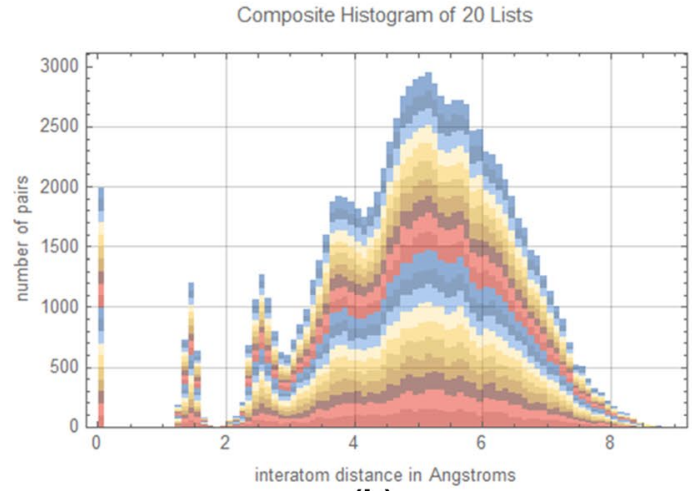

(b)

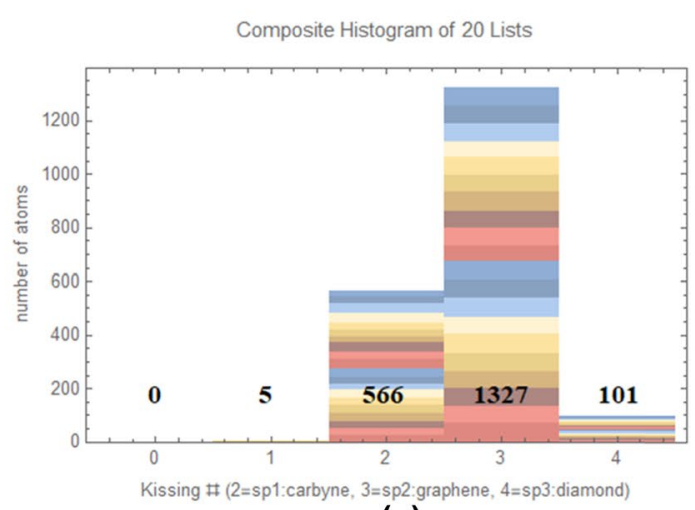

(c)

Fig. 3 Complete analysis report for 100 atom sets. (a) Total loop count, (b) composite interatom spacing histogram and (c) composite coordination number plot

\section{Results}

Figure 2 illustrates one way to visualize interatom covalent connections (here associated with the distinct class of interatom distances smaller than $1.7 \AA$ ) using a 13 atom set for clarity. Bonds that cross supercell boundaries are shown only once, and the atoms outside the central volume are un-numbered.

Figure 3 provides a complete analysis report from 100 atom sets that includes the total loop count table (Fig. 3a), the composite interatom spacing histogram (Fig. 3b) and the composite coordination number plot (Fig. 3c). Figure 3a lists the number of loops found for each type $[n=3$ (triangles) to 7 (heptagons)]. Although graph theory can easily identify all $n$-atom loops, e.g., with bond length shorter than say $1.7 \AA$, a subset of these are "spanning loops", i.e., which return not to the starting atom but to a periodic instance of that starting atom. Topological loops also include "super loops", for instance the hexagon associated with a pentagon and triangle sharing a common side. Primitive loops are the ones which cannot break further into smaller loops. In our loop analyses, we count only non-spanning primitive loops using standard algorithms [19]. Figure $3 \mathrm{~b}$ shows the histogram of distances between each atom and Fig. $3 \mathrm{c}$ lists the total number of atoms that contain specific coordination. Figures $3 b$, c track list identities using different colors for each. In Fig. 3c, in each bar, the total number of atoms is marked for each coordination. For example, 566 atoms have 2-fold ( $\mathrm{sp}$ ) coordination in this list.

Interatom distance histograms (available in Supplementary Material Sect. 1) exhibited an interatom distance abundance gap between 1.7 and $2 \AA$, so that (in agreement with the literature) we categorize pair interactions for spacings less than $1.7 \AA$ as "covalent", but those higher than $2 \AA$ are listed as "non-covalent" interactions.
Table 1 Total coordination numbers for all relaxations

\begin{tabular}{lllcrc}
\hline Coordination number & 0 & $1=\mathrm{sp}^{0}$ & $2=\mathrm{sp}^{1}$ & $3=\mathrm{sp}^{2}$ & $4=\mathrm{sp}^{3}$ \\
\hline 13 Atom sets & 0 & 0 & 100 & 154 & 3 \\
20 Atom sets & 0 & 1 & 91 & 289 & 19 \\
30 Atom sets & 0 & 5 & 168 & 397 & 30 \\
40 Atom sets & 1 & 1 & 225 & 535 & 38 \\
60 Atom sets & 0 & 1 & 314 & 841 & 44 \\
100 Atom sets & 0 & 5 & 566 & 1327 & 101 \\
\hline
\end{tabular}

\section{Fraction of coordination (per atom) as a percentage} vs. Coordination number

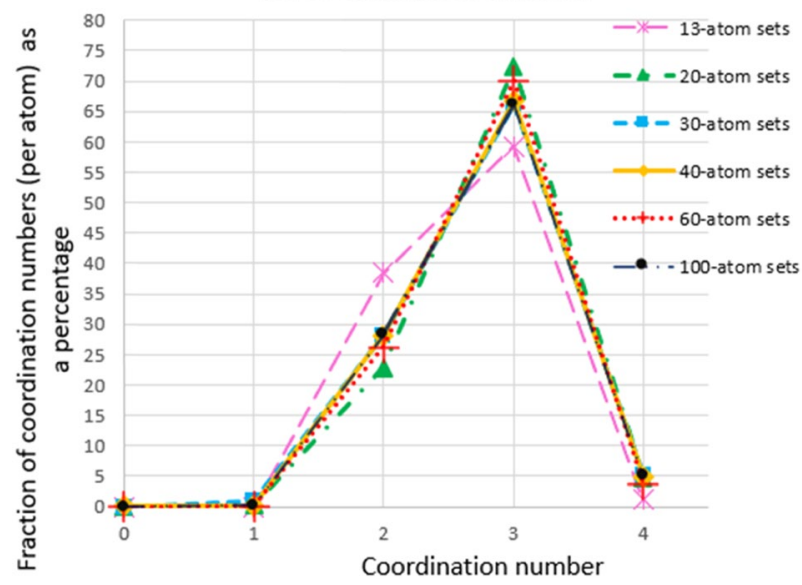

Fig. 4 Fraction of coordination numbers per atom as a percentage (\%) vs. coordination number

Table 1 lists coordination numbers, and Fig. 4 is coordination number percentages, for all relaxation reports. Most atoms have covalent coordination number 3 or 2 at this density. 
Table 2 Total loop counts for all relaxations

\begin{tabular}{lcclcc}
\hline Loop type & 3-Loop & 4-Loop & 5-Loop & 6-Loop & 7-Loop \\
\hline 13 Atom sets & 11 & 6 & 12 & 6 & 6 \\
20 Atom sets & 23 & 10 & 26 & 14 & 18 \\
30 Atom sets & 25 & 18 & 27 & 15 & 20 \\
40 Atom sets & 47 & 27 & 27 & 22 & 33 \\
60 Atom sets & 45 & 39 & 50 & 39 & 56 \\
100 Atom sets & 100 & 50 & 92 & 48 & 89 \\
\hline
\end{tabular}

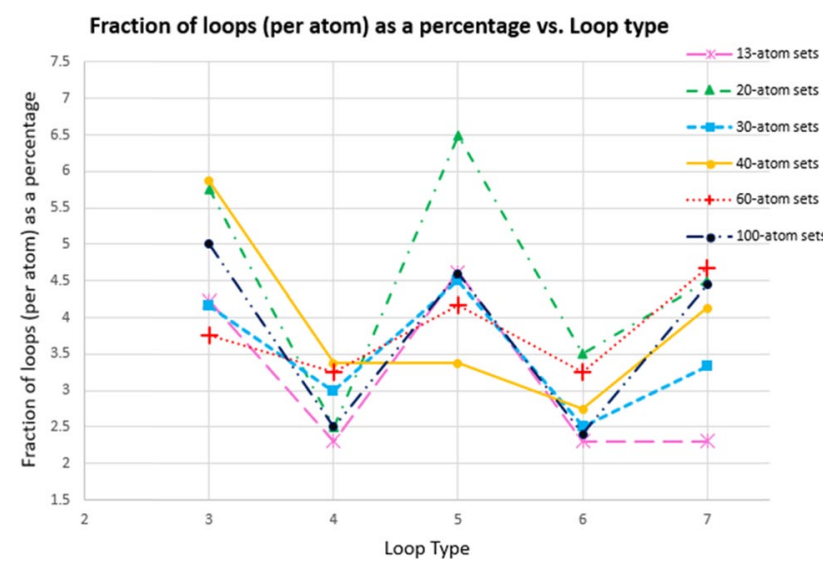

Fig. 5 Fraction of loops (per atom) as a percentage (\%) vs. loop type

Table 2 lists loop counts, and Fig. 5 loops per atom, for loops with 3 to 7 atoms. DFT relaxation predict abundant 3 loops, consistent with earlier cited reports [20]. Three and four atom loops are not experimentally abundant in graphene sheet structures [12, 21, 22]. At this density, 5 loops (common experimentally, e.g., in fullerenes) are generally more abundant than 6 atom loops. Loops with 7 or more atoms are also abundant in simulations [20], and 7 loops (combined with a 5-loop) are even used to change the diameter of carbon nanotubes. However, the saddle-like bend that a 7-loop introduces in a graphene sheet, unlike the conical-bend of a 5-loop, are not consistent with our observation of edge-on sheet intersection angles in the presolar cores.

\section{Discussion}

The interatom distance abundance gap between 1.7 and 2 $\AA$, helps to justify the practice (common in the literature) of identifying atom-pair separations less than $1.7 \AA$ as "covalent bonds". Interatom distances higher than $2 \AA$ are by default then associated with non-covalent (with coordination numbers up to 12) expected in a quenched liquid.

Most list atoms after " $0 \mathrm{~K}$ " relaxation had covalent coordination 2 or 3 . This agrees with previous studies as our density is considered "intermediate" and expected to show a mixture of 2 and 3- with 4-fold coordination under 5\% [20, 23-26].

We also compare absolute number of 5- and 6 loops per carbon atom, with experimental data on the number of graphene sheets per carbon atom present in the cores of graphite-rimmed carbon spheres condensed in the atmosphere of red giant stars, and in core-only carbon spheres condense in our laboratory. The latter data come from powder diffraction measurements of graphene sheet coherence widths (sizes) and the fraction of carbon atoms "crystallized" into graphene sheets [12]. If such loops seeded the nucleation and growth of those graphene sheets, the comparison provides us with estimates of the survival fraction of such loops during the subsequent crystallization process in both environments. That, powder diffraction data suggests that presolar specimens are $40 \%$ (by mass) graphene sheets typically 600 atoms in size (i.e., from a $40 \AA$ coherence width), while lab-grown specimens are $12 \%$ graphene sheets typically 60 atoms in size (i.e., from a $12.6 \AA$ coherence width), with the remainder of the carbon atoms disordered. Given $9 \times 10^{22}$ carbon atom $/ \mathrm{cm}^{3}$ in carbon of $1.8 \mathrm{~g} / \mathrm{cm}^{3}$ density, this suggests $6 \times 10^{19}$ sheets $/ \mathrm{cm}^{3}$ in the presolar specimens, and $1.8 \times 10^{20}$ smaller sheets $/ \mathrm{cm}^{3}$ in the lab-grown specimens.

If each of these sheets was nucleated on a single 6-loop, then we require this many 6 loops $/ \mathrm{cm}^{3}$ to have survived growth to 600 atom size. Since 5 loops each nucleate as many as 5 such sheets, only one fifth as many "fully grown" 5 loops would be needed to seed the same number for flat sheets. The implications of these model results, considering the final sheet size and fraction-crystalline observations reported here [12], are summarized in Table 3.

Table 3 Fraction-crystalline observations

\begin{tabular}{lllllll}
\hline Seed type & $\begin{array}{l}\text { Loops in a VASP } \\
\text { ab initio local minimum }\end{array}$ & $\begin{array}{l}\text { Sheets seeded } \\
\text { per loop }\end{array}$ & $\begin{array}{l}\text { Lab condensed abun- } \\
\text { dance of grown seeds }\end{array}$ & $\begin{array}{l}\text { \% Growing to 60 } \\
\text { atoms in the lab }\end{array}$ & $\begin{array}{l}\text { Presolar abundance } \\
\text { of grown seeds }\end{array}$ & $\begin{array}{l}\% \text { Growing } \\
\text { to } 600 \text { atoms } \\
\text { presolar }\end{array}$ \\
\hline 6 Loops & $2.6 \times 10^{21} / \mathrm{cm}^{3}$ & 1 & $1.8 \times 10^{20} / \mathrm{cm}^{3}$ & 6.9 & $6.0 \times 10^{19} / \mathrm{cm}^{3}$ & 2.3 \\
5 Loops & $5.0 \times 10^{21} / \mathrm{cm}^{3}$ & 5 & $3.6 \times 10^{19} / \mathrm{cm}^{3}$ & 0.72 & $1.2 \times 10^{19} / \mathrm{cm}^{3}$ & 0.24 \\
\hline
\end{tabular}


This suggests that only about a 10th as many "successful" 5 loops seeds is needed (in both types of specimen) to overtake the contribution from "successful" 6-loop seeds. It also suggests that a useful nucleation and growth model for unlayered graphene in a cooling carbon melt should predict that only a third as many sheets will survive in the slower growth to 600 atoms, than in the few millisecond growths to 60 atoms in our laboratory. In context of such a model, we might be able to learn something about (i) the cooling rate of carbon droplets condensed around red giant stars, and well as (ii) the conditions needed to synthesize comparable material (with its promising diffusion barrier properties) in the laboratory.

Figure 6 shows 5-/6-loop ratio against the total number of atoms ' $n$ ' in the simulation cell. It compares the data with a constant model, plus two ad hoc-models in which 5/6 ratio decreases with $n$, to see if our high $5 / 6$ ratio is an artifact of the small number of atoms in our sets.

The two-parameter model signifies that the loop ratio is a linear function of the ratio between the number of ways to create 5- and 6-loop in a set of $n$ atoms, while the 3-parameter model assumes a quadratic dependence instead. Statistical uncertainties are smaller for larger $n$-sets, and model selection measures 'Akaike/Bayesian (AIC/BIC). Information Criteria of 7.77/7.35 (constant model), compared to 8.93/8.31 (2-parameter), and 11.4/10.6 (3-parameter) in weighted fits argue against a significant decrease in 5/6 ratio with increasing number of atoms in the set.

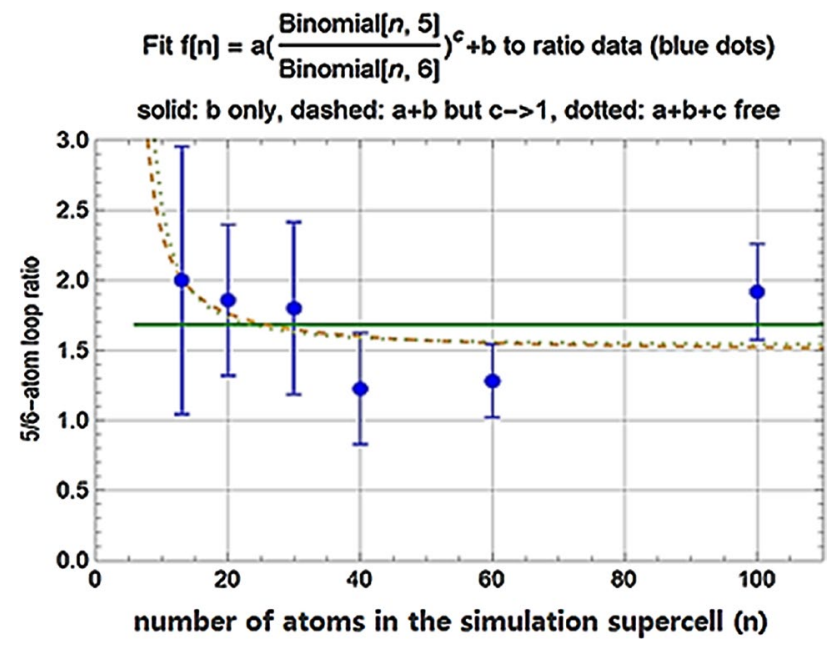

Fig. 6 5-/6-Loop ratios vs. $n$, solid green: constant model, dashed: two-parameter model, dotted: three-parameter model

\section{Conclusion}

Presolar and lab-grown specimens contain interesting spheres made of unlayered graphene in a solid matrix, which may open the door to low pressure studies of liquid carbon. DFT-VASP supercell relaxation of random carbon atom clusters in the observed density range agree with existing literature and suggest that 5-/6-loop abundance ratios around one are not an artifact of the number of atoms in the simulation. The number of loops seen in our simulations, per carbon atom, is also greater than the number of sheets per carbon atom inferred from diffraction observations on both presolar and lab-grown cores [10, 12]. Therefore, the nucleation of graphene sheets on pentagonal loops might help explain the intersecting line segments seen in HRTEM images of presolar cores [9].

Separate molecular dynamics and nucleation growth modeling is still needed to see if indeed the 5 loops compete effectively with 6 loops in nucleating the larger observed structures. Separately, studies of the diffusion barrier properties of randomly oriented sheets in a disordered matrix, oven design to provide greater control of cooling rate, and supercooling thresholds for containerless carbon liquid, may help with materials science and astrophysical applications.

Acknowledgments The VASP calculations, and manuscript writing, were done by CS, the LCBOP calculations were provided by PC, the data on presolar and lab-grown specimens as well as some Mathematica programs were provided by PF. A special thanks to Dr. Eric Majzoub who helped provide the access and supervised the VASP code, MST-Rolla for the access to the Forge cluster and UMSL department of Physics and Astronomy for a place to work.

Data availability The corresponding author confirm that the all data used for finding these new findings (results) are available in the original article and in the Supplementary Material attached with the original article.

\section{Compliance with ethical standards}

Conflict of interest On behalf of all authors, the corresponding author declares that there is no conflict of interest.

Open Access This article is licensed under a Creative Commons Attribution 4.0 International License, which permits use, sharing, adaptation, distribution and reproduction in any medium or format, as long as you give appropriate credit to the original author(s) and the source, provide a link to the Creative Commons licence, and indicate if changes were made. The images or other third party material in this article are included in the article's Creative Commons licence, unless indicated otherwise in a credit line to the material. If material is not included in the article's Creative Commons licence and your intended use is not permitted by statutory regulation or exceeds the permitted use, you will need to obtain permission directly from the copyright holder. To view a copy of this licence, visit http://creativecommons.org/licenses/by/4.0/. 


\section{References}

1. P. Fraundorf, D.E. Brownlee, R.M. Walker, Laboratory studies of interplanetary dust, in Comets. ed. by L. Wilkening (University of Arizona Press, Tucson, 1982), pp. 383-409

2. J.P. Bradley, D.E. Brownlee, P. Fraundorf, Science 226, 1432 1434 (1984)

3. T. Bernatowicz, G. Fraundorf, T. Ming, E. Anders, B. Wopenka, E. Zinner, P. Fraundorf, Nature 330, 728-730 (1987)

4. E. Zinner, Annu. Rev. Earth Planet. Sci. 26, 147-188 (1998)

5. T. Bernatowicz, R.M. Walker, Phys. Today 50, 26 (1997)

6. L.R. Nittler, F. Ciesla, Annu. Rev. Astron. Astrophys. 54, 53-93 (2016)

7. D. Kasuya, M. Yudasaka, K. Takahashi, F. Kokai, S. Iijima, J. Phys. Chem. B 106, 4947-4951 (2002)

8. W.A. De Heer, P. Poncharal, C. Berger, J. Gezo, Z. Song, J. Bettini, D. Ugarte, Science 307(5711), 907-910 (2005)

9. P. Fraundorf, M. Wackenhut, Astrophys. J. Lett. 578(2), L153L156 (2002)

10. P. Fraundorf, T. Hundley, M. Lipp, HAL-02238804 (2019)

11. T.J. Hundley, P. Fraundorf, in 49th Lunar and Planetary Science Conference 2018 (LPI Contribution No. 2083), pp. 2154-2155 (2018)

12. P. Fraundorf, M. Lipp, T. Hundley, C. Silva, P. Chrostoski, Microsc. Microanal. 26(S2), 2838-2840 (2020)
13. P. Fraundorf, S. Chathuri, P. Chrostoski, M. Lipp, COSPAR 2021 Sydney (International Council of Scientific Unions Committee on Space Research, 2021, in prep)

14. T. Bernatowicz, R. Cowsik, P.C. Gibbons, K. Lodders, B. Fegley Jr., S. Amari, R.S. Lewis, Astrophys. J. 472, 760 (1996)

15. P. Fraundorf, K. Pisane, E. Mandell, R. Collins, Microsc. Microanal. 16(Supplement 2), 1534-1535 (2010)

16. P.E. Blöchl, Phys. Rev. B 50, 17953 (1994)

17. J.P. Perdew, K. Burke, M. Ernzerhof, Phys. Rev. Lett. 77, 3865 (1996)

18. J.H. Los, A. Fasolino, Phys. Rev. B 68, 024107 (2003)

19. D.S. Franzblau, Phys. Rev. 44, 4925 (1991)

20. V.L. Deringer, G. Csányi, Phys. Rev. B 95, 094203 (2017)

21. C. Toh, H. Zhang, J. Lin, A.S. Mayorov, Y. Wang, C.M. Orofeo et al., Nature 577, 199-203 (2020)

22. M.S. Dresselhaus, G. Dresselhaus, P.C. Eklund, Science of Fullerenes and Carbon Nanotubes (Academic, San Diego, 1996).

23. C. Cannella, N. Goldman, J. Phys. Chem. 119, 29-31 (2015)

24. J.R. Morris, C.Z. Wang, K.M. Ho, Phys. Rev. B 52, 4138 (1995)

25. C.J. Wu, J.N. Glosli, G. Galli, F.H. Ree, Phys. Rev. Lett. 89, 135701 (2002)

26. N.A. Marks, N.C. Cooper, D.R. McKenzie, Phys. Rev. B 65, $075411(2002)$ 